\title{
Complete genome sequence of Desulfohalobium retbaense type strain $\left(\mathrm{HR}_{100}{ }^{\top}\right)$
}

\author{
Stefan Spring1 , Matt Nolan², Alla Lapidus², Tijana Glavina Del Rio², Alex Copeland², Hope \\ Tice $^{2}$, Jan-Fang Cheng ${ }^{2}$, Susan Lucas ${ }^{2}$, Miriam Land ${ }^{2,5}$, Feng Chen², David Bruce ${ }^{2,3}$, Lynne \\ Goodwin ${ }^{2,3}$, Sam Pitluck², Natalia Ivanova², Konstantinos Mavromatis', Natalia Mikhailova², \\ Amrita Pati ${ }^{2}$, Amy Chen ${ }^{4}$, Krishna Palaniappan ${ }^{4}$, Loren Hauser ${ }^{2,5}$, Yun-Juan Chang ${ }^{2,5}$, Cynthia \\ D. Jeffries ${ }^{2,5}$, Christine Munk ${ }^{3}$, Hajnalka Kiss ${ }^{2,3}$, Patrick Chain²,3, Cliff Han ${ }^{2,3}$, Thomas Bret- \\ tin $^{2,3}$, John C. Detter ${ }^{2,3}$, Esther Schüler ${ }^{1}$, Markus Göker ${ }^{1}$, Manfred Rohde $^{7}$, Jim Bristow ${ }^{2}$, Jo- \\ nathan A. Eisen ${ }^{2,6}$, Victor Markowitz ${ }^{8}$, Philip Hugenholtz ${ }^{2}$, Nikos C. Kyrpides ${ }^{2}$, and Hans- \\ Peter Klenk ${ }^{1 *}$ \\ ${ }^{1}$ DSMZ - German Collection of Microorganisms and Cell Cultures GmbH, Braunschweig, \\ Germany \\ ${ }^{2}$ DOE Joint Genome Institute, Walnut Creek, California, USA \\ ${ }^{3}$ Los Alamos National Laboratory, Bioscience Division, Los Alamos, New Mexico, USA \\ ${ }^{4}$ Lawrence Livermore National Laboratory, Livermore, California, USA \\ ${ }^{5}$ Oak Ridge National Laboratory, Oak Ridge, Tennessee, USA \\ ${ }^{6}$ University of California Davis Genome Center, Davis, California, USA \\ ${ }^{7}$ HZI - Helmholtz Centre for Infection Research, Braunschweig, Germany \\ ${ }^{8}$ Biological Data Management and Technology Center, Lawrence Berkeley National \\ Laboratory, Berkeley, California, USA
}

*Corresponding author: Hans-Peter Klenk

Keywords: sulfate-reducer, Gram-negative, mesophile, moderately halophilic, strictly anaerobic, hydrogen utilization, hypersaline lake, Desulfohalobiaceae, Deltaproteobacteria, Proteobacteria, GEBA

Desulfohalobium retbaense (Ollivier et al. 1991) is the type species of the polyphyletic genus Desulfohalobium, which comprises, at the time of writing, two species and represents the family Desulfohalobiaceae within the Deltaproteobacteria. D. retbaense is a moderately halophilic sulfate-reducing bacterium, which can utilize $\mathrm{H}_{2}$ and a limited range of organic substrates, which are incompletely oxidized to acetate and $\mathrm{CO}_{2}$, for growth. The type strain $\mathrm{HR}_{100}{ }^{\top}$ was isolated from sediments of the hypersaline Retba Lake in Senegal. Here we describe the features of this organism, together with the complete genome sequence and annotation. This is the first completed genome sequence of a member of the family Desulfohalobiaceae. The 2,909,567 bp genome (one chromosome and a 45,263 bp plasmid) with its 2,552 protein-coding and 57 RNA genes is a part of the Genomic Encyclopedia of Bacteria and Archaea project.

\section{Introduction}

Strain $\mathrm{HR}_{100^{\mathrm{T}}}$ (= DSM 5692) is the type strain of the species Desulfohalobium retbaense [1]. $\mathrm{HR}_{100^{\mathrm{T}}}$ is the only strain available from culture collections belonging to this species and was isolated from surface sediments of the hypersaline Retba Lake in Senegal (Western Africa). This strain was the first cultivated sulfate-reducing bacterium, which grows in media containing $\mathrm{NaCl}$ concentrations up to $24 \%$ and the first described hydrogenotrophic anaerobe able to grow at salinities above $10 \%[1]$.
Interestingly, the total salt concentration of the Retba Lake was $34 \%$ at the time of sampling, which would indicate that cells of this strain were not able to proliferate in the habitat from which they were originally isolated. This phenomenon was later also reported in a study on the diversity of sulfate-reducing bacteria in hypersaline sediments of the Great Salt Lake (Utah) [2]. This effect could either be explained by niches of lower salinity in the respective habitats, which would allow 
proliferation at distinct sites or, alternatively, that the in vitro halotolerance of these strains is different from the salt tolerance in the natural environment. One reason for the observed growth inhibition of sulfate-reducers at salinities above $24 \%$ may be the energy expensive synthesis of compatible osmotic solutes, which are required in large amounts to retain cellular integrity at high external salt concentrations. Under anoxic conditions bacteria that depend on sulfate as electron acceptor gain less energy than microorganisms that use photosynthesis or denitrification for growth, so that the latter metabolic types have a selective advantage in hypersaline environments [3]. Here we present a summary classification and a set of features for $D$. retbaense strain $\mathrm{HR}_{100^{\mathrm{T}}}$, together with the description of the complete genomic sequencing and annotation.

\section{Classification and features}

So far, no $16 \mathrm{~S}$ rRNA gene sequences with high similarity ( $>95 \%$ ) to the sequence of $D$. retbaense have been deposited in public databases, although several anoxic sediments with high salinity have been analyzed by cultivation independent methods (as of October 2009) since D. retbaense was described. Consequently, it appears that cells of sulfate-reducing bacteria related to this species are of very low abundance in most hypersaline environments. Besides several strains of the genus
Desulfovibrio, the only other member of the order Desulfovibrionales with a sequenced genome is Desulfomicrobium baculatum type strain $\mathrm{X}^{\mathrm{T}}$ [4].

D. retbaense is the type species of the genus Desulfohalobium, which represents the recently proposed family Desulfohalobiaceae within the class Deltaproteobacteria [5]. The genus Desulfohalobium is currently polyphyletic due to the species D. utahense, which is phylogenetically more closely related to Desulfovermiculus halophilus, with high bootstrapping support in the 16S rRNA tree (Figure 1) Also, both share a 16S rRNA gene sequence similarity of $96.9 \%$, whereas the two $D e$ sulfohalobium species display a sequence similarity of only $90.5 \%$. Hence, it is possible that the species $D$. utahense has been misclassified, although it appears to be phenotypically more similar to $D$. retbaense than to Desulfovermiculus halophilus [10]. The taxonomy of the two genera thus needs to be reconsidered.

Figure 1 shows the phylogenetic neighborhood of D. retbaense strain $\mathrm{HR}_{100}{ }^{\mathrm{T}}$ in a $16 \mathrm{~S}$ rRNA based tree. The two 16S rRNA gene copies in the genome of strain $\mathrm{HR}_{100}{ }^{\mathrm{T}}$ do not differ from each other, and differ by four nucleotides from the previously published 16S rRNA sequence generated from DSM 5692 (X99235). The difference between the genome data and the reported 16S rRNA gene sequence is most likely due to sequencing errors in the previously reported sequence data.

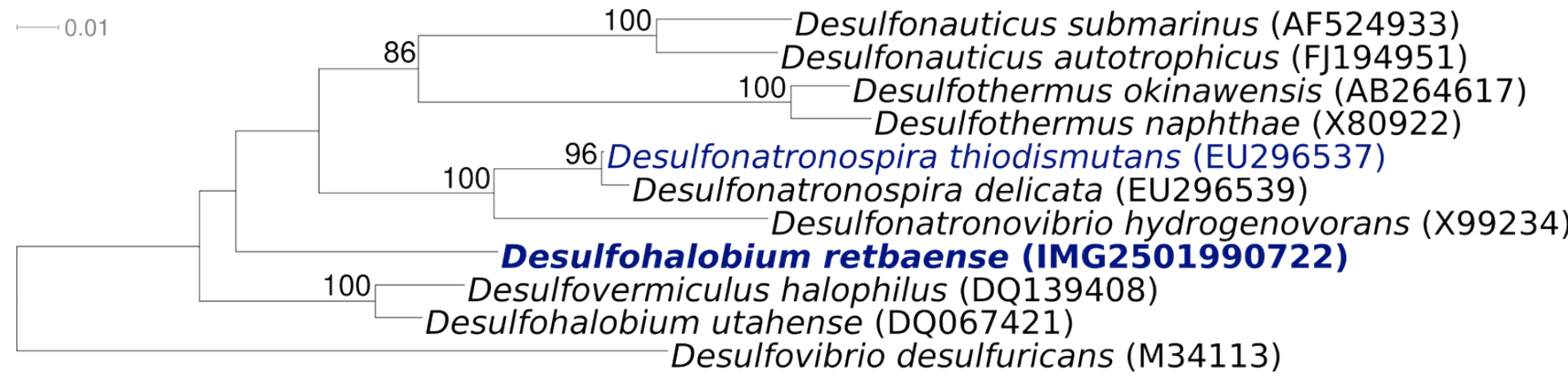

Figure 1. Phylogenetic tree highlighting the position of strain $\mathrm{HR}_{100}{ }^{\top}, D$. retbaense DSM 5692, relative to the other type strains within the family. The tree was inferred from 1,386 aligned characters [6,7] of the 16S rRNA gene sequence under the maximum likelihood criterion [8] and rooted in accordance with the type strain of the order Desulfovibrionales. The branches are scaled in terms of the expected number of substitutions per site. Numbers above branches are support values from 1,000 bootstrap replicates if larger than 60\%. Lineages with type strain genome sequencing projects registered in GOLD [9] are shown in blue, published genomes in bold.

Cells of D. retbaense $\mathrm{HR}_{100}{ }^{\mathrm{T}}$ are straight to slightly curved rods with rounded ends (Table 1 and Figure 2). They have dimensions of 0.7-0.9 x 1-3 $\mu \mathrm{m}$ and stain Gram-negative. In medium containing lactate as substrate, cells can form filaments up to $20 \mu \mathrm{m}$ in length. Motility is conferred by one or two polar flagella [1]. 
Spring et al.

Strain $\mathrm{HR}_{100^{\mathrm{T}}}$ is halophilic and requires $\mathrm{NaCl}$ and $\mathrm{MgCl}_{2}$ for growth. The optimal $\mathrm{NaCl}$ concentration for growth is near $10 \%$ and salinities up to $24 \%$ are tolerated. The $\mathrm{pH}$ range for growth is 5.5 to 8.0 with an optimum between pH 6.5 and 7.0. Growth of this strain occurs at temperatures from 25 to $43^{\circ} \mathrm{C}$ and is optimal between 37 and $40^{\circ} \mathrm{C}$ [1].

The nutritional characteristics of strain $\mathrm{HR}_{100}{ }^{\mathrm{T}}$ are as follows: Vitamins and an organic carbon source are required for growth in mineral medium. Hydrogen is utilized mixotrophically with acetate, yeast extract or biotrypcase as the carbon source, but not autotrophically. Organic carbon sources supporting growth are formate, ethanol, pyruvate and lactate. Sulfate, sulfur, thiosulfate and sulfite are used as electron acceptors and are reduced to $\mathrm{H}_{2} \mathrm{~S}$. In the absence of sulfate pyruvate can be also utilized fermentatively [1].

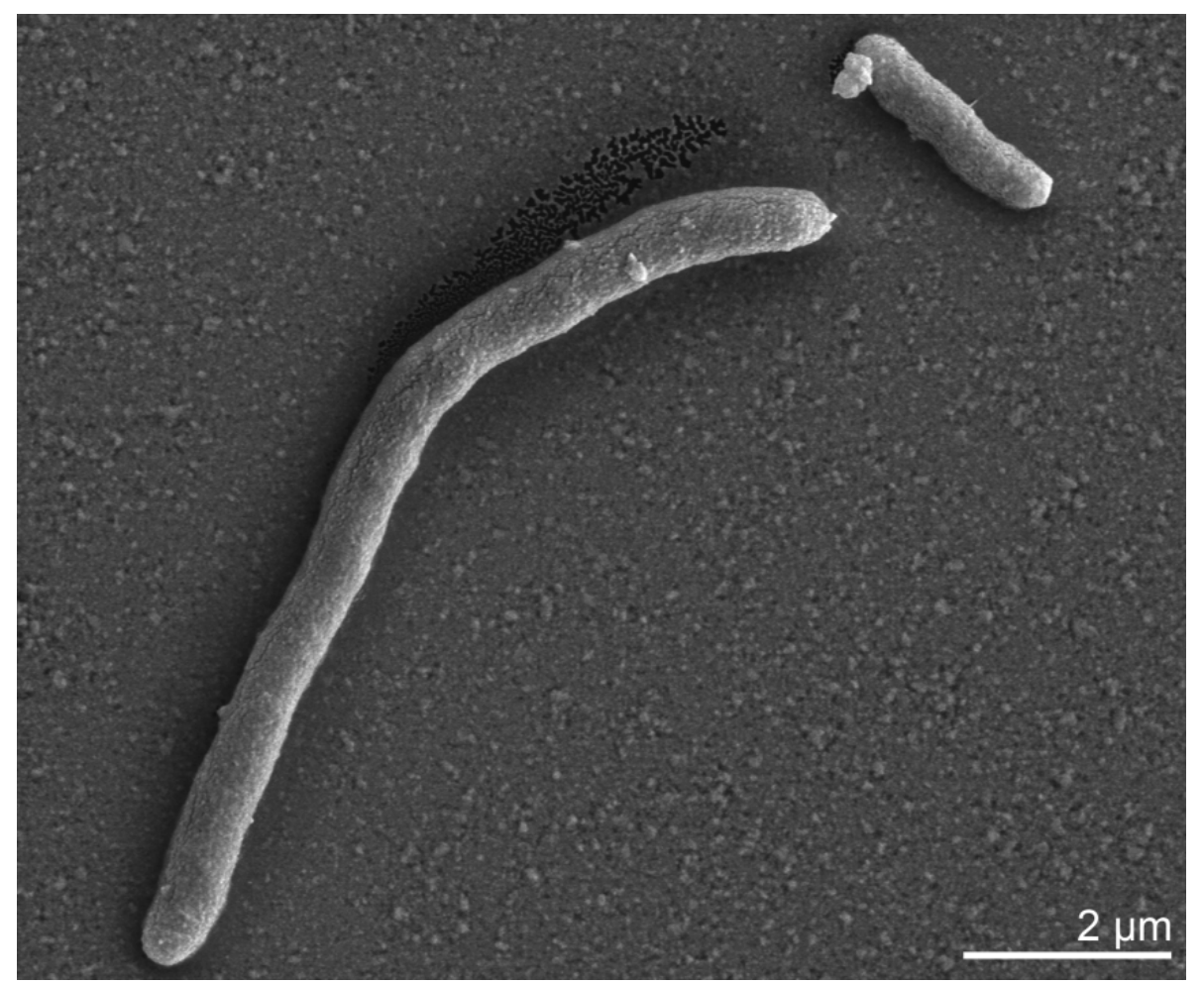

Figure 2. Scanning electron micrograph of cells of $D$. retbaense strain $\mathrm{HR}_{100}{ }^{\mathrm{T}}$

\section{Chemotaxonomy}

Spectrophotometry of cell extracts indicate the presence of soluble $c$-type cytochromes having absorption maxima at 418.5, 522.5 and $552 \mathrm{~nm}$ in the reduced state, which would be characteristic for cytochrome $c_{3}$. A dissimilatory sulfite reductase with a similar absorption spectrum as the enzyme of Desulfomicrobium baculatum (desulforubidin) was detected, but no desulfoviridin, which is diagnostic for members of the genus Desulfovibrio [1]. The respiratory lipoquinone composition of strain $\mathrm{HR}_{100^{\mathrm{T}}}$ has not been reported, but the moderately related species Desulfovermiculus halophilus was shown to contain the menaquinone MK-7 [18]. The whole cell fatty acid pattern of strain $\mathrm{HR}_{100^{T}}$ is dominated by straight- and branched-chain saturated fatty acids (approx. $68 \%$ ). Branched chain saturated fatty acids ac- count for $30 \%$ of the total fatty acids, with iso- $\mathrm{C}_{15: 0}$ predominating. In addition, the fatty acid profile contains branched-chain, mono-unsaturated fatty acids, such as iso- $\mathrm{C}_{17: 1 \omega 7 \mathrm{c}}$ and branched $\mathrm{C}_{18: 1 \omega} 6$ [1].

\section{Genome sequencing and annotation Genome project history}

This organism was selected for sequencing on the basis of its phylogenetic position, and is part of the Genomic Encyclopedia of Bacteria and Archaea project [19]. The genome project is deposited in the Genomes OnLine Database [9] and the complete genome sequence is available in GenBank. Sequencing, finishing and annotation were performed by the DOE Joint Genome Institute (JGI). A summary of the project information is shown in Table 2. 
Table 1. Classification and general features of $D$. retbaense strain $\mathrm{HR}_{100}{ }^{\top}$ according to the MIGS recommendations [11]

\begin{tabular}{|c|c|c|c|}
\hline MIGS ID & Property & Term & Evidence code \\
\hline & \multirow{8}{*}{ Current classification } & Domain Bacteria & TAS [12] \\
\hline & & Phylum Proteobacteria & TAS [13] \\
\hline & & Class Deltaproteobacteria & TAS $[14,15]$ \\
\hline & & Order Desulfovibrionales & TAS [14] \\
\hline & & Family Desulfohalobiaceae & TAS [14] \\
\hline & & Genus Desulfohalobium & TAS [1] \\
\hline & & Species Desulfohalobium retbaense & TAS [1] \\
\hline & & Type strain $\mathrm{HR}_{100}$ & TAS [1] \\
\hline & Gram stain & negative & TAS [1] \\
\hline & Cell shape & rod with rounded ends & TAS [1] \\
\hline & Motility & motile (one or two polar flagella) & TAS [1] \\
\hline & Sporulation & nonsporulating & TAS [1] \\
\hline & Temperature range & $25-43^{\circ} \mathrm{C}$ & TAS [1] \\
\hline & Optimum temperature & $37-40^{\circ} \mathrm{C}$ & TAS [1] \\
\hline & Salinity & $>0-240 \mathrm{~g} / \mathrm{l}$ (optimum $100 \mathrm{~g} / \mathrm{l}$ ) & TAS [1] \\
\hline \multirow[t]{3}{*}{ MIGS-22 } & Oxygen requirement & obligate anaerobic & TAS [1] \\
\hline & Carbon source & acetate, biotrypcase, yeast extract & TAS [1] \\
\hline & Energy source & $\mathrm{H}_{2}$, formate, lactate, ethanol, pyruvate & TAS [1] \\
\hline MIGS-6 & Habitat & hypersaline sediments & TAS [1] \\
\hline MIGS-15 & Biotic relationship & free living & NAS \\
\hline \multirow[t]{3}{*}{ MIGS-14 } & Pathogenicity & none & TAS [16] \\
\hline & Biosafety level & 1 & TAS [16] \\
\hline & Isolation & surface sediment & TAS [1] \\
\hline MIGS-4 & Geographic location & Retba Lake, Senegal & TAS [1] \\
\hline MIGS-5 & Sample collection time & 1989 & NAS \\
\hline MIGS-4.1 & Latitude, Longitude & $14.84,-17.23$ & NAS \\
\hline MIGS-4.2 & & & \\
\hline MIGS-4.3 & Depth & not reported & \\
\hline MIGS-4.4 & Altitude & $-4 \mathrm{~m}$ & TAS [1] \\
\hline
\end{tabular}

Evidence codes - IDA: Inferred from Direct Assay (first time in publication); TAS: Traceable Author Statement (i.e., a direct report exists in the literature); NAS: Non-traceable Author Statement (i.e., not directly observed for the living, isolated sample, but based on a generally accepted property for the species, or anecdotal evidence). These evidence codes are from the Gene Ontology project [17]. If the evidence code is IDA, then the property was directly observed for a live isolate by one of the authors or an expert mentioned in the acknowledgments.

\section{Growth conditions and DNA isolation}

D. retbaense strain $\mathrm{HR}_{100^{\mathrm{T}}}$, DSM 5692, was grown anaerobically in DSMZ medium 499 [20] at $35^{\circ} \mathrm{C}$. DNA was isolated from 1-1.5 g of cell paste using Qiagen Genomic 500 DNA Kit (Qiagen, Hilden, Germany) following the manufacturer's instructions.

\section{Genome sequencing and assembly}

The genome was sequenced using a combination of $8 \mathrm{~kb}$ and fosmid DNA libraries. All general aspects of library construction and sequencing performed at the JGI can be found at the http://www.jgi.doe.gov/. The Phred/Phrap/Consed software package (http://www.phrap.com) was used for sequence assembly and quality assessment. Possible mis-assemblies were corrected with Dupfinisher [21] or transposon bombing of bridging clones [22]. Gaps between contigs were closed by editing in Consed, custom primer walk or PCR amplification. Sanger finishing reads $(\mathrm{n}=889)$ were produced to close gaps and to raise the quality of the finished sequence. The error rate of the completed genome sequence is less than 1 in 100,000 . The final assembly consists of 42,114 Sanger reads. Together all sequence provided $10.7 \times$ coverage of the genome. 
Table 2. Genome sequencing project information

\begin{tabular}{|c|c|c|}
\hline MIGS ID & Property & Term \\
\hline MIGS-31 & Finishing quality & Finished \\
\hline MIGS-28 & Libraries used & $\begin{array}{l}\text { Two genomic libraries - } 8 \mathrm{~kb} \text { pMCL200 } \\
\text { and fosmid pcc1Fos }\end{array}$ \\
\hline MIGS-29 & Sequencing platforms & $\mathrm{ABI} 3730$ \\
\hline MIGS-31.2 & Sequencing coverage & $10.7 \times$ Sanger \\
\hline MIGS-30 & Assemblers & phrap \\
\hline \multirow[t]{6}{*}{ MIGS-32 } & Gene calling method & Prodigal \\
\hline & INSDC ID & $\begin{array}{l}\text { CP001734 (chromosome) } \\
\text { CP001735 (plasmid) }\end{array}$ \\
\hline & GenBank Date of Release & September 14, 2009 \\
\hline & GOLD ID & Gc01111 \\
\hline & NCBI project ID & 29199 \\
\hline & Database: IMG-GEBA & 2501939614 \\
\hline \multirow[t]{2}{*}{ MIGS-13 } & Source material identifier & DSM 5692 \\
\hline & Project relevance & Tree of Life, GEBA \\
\hline
\end{tabular}

\section{Genome annotation}

Genes were identified using Prodigal [23] as part of the Oak Ridge National Laboratory genome annotation pipeline, followed by a round of manual curation using the JGI GenePRIMP pipeline [24]. The predicted CDSs were translated and used to search the National Center for Biotechnology Information (NCBI) nonredundant database, UniProt, TIGRFam, Pfam, PRIAM, KEGG, COG, and InterPro databases. Additional gene prediction analysis and manual functional annotation was performed within the Integrated Microbial Genomes Expert Review (IMG-ER) platform [25].

\section{Genome properties}

The 2,909,567 bp genome consists of a 2,864,304 bp long chromosome and a 45,263 bp long plasmid with a $57.3 \%$ GC content (Table 3 and Figure $3)$. Of the 2,609 genes predicted, 2,552 were protein coding genes, and 57 RNAs; 29 pseudogenes were also identified. The majority of the proteincoding genes $(73.6 \%)$ were assigned with a putative function while those remaining were annotated as hypothetical proteins. The distribution of genes into COGs functional categories is presented in Table 4.

\section{Insights from the genome sequence Electron donor utilization}

Similar to representatives of the genus Desulfovibrio, the preferred substrates of $D$. retbaense are $\mathrm{H}_{2}$ and lactate, the latter which is incompletely oxidized to acetate. Several genes could be identi- fied that are involved in $\mathrm{H}_{2}$ dependent sulfate reduction in Desulfovibrio species. It is assumed that in species of this genus $\mathrm{H}_{2}$ is oxidized by periplasmic Fe- or NiFeSe-hydrogenases and the resulting electrons are transferred to a pool of periplasmic cytochrome $c_{3}$. Then, membrane-bound protein complexes transfer electrons from the pool of reduced cytochrome $c_{3}$ to menaquinone or directly to cytoplasmic enzymes involved in the reduction of sulfate to sulfide [26]. Recently, a novel molybdopterin oxidoreductase (Mop) could be identified in Desulfovibrio desulfuricans G20 that may represent a periplasm-facing transmembrane complex, which shuttles electrons from cytochrome $c_{3}$ to the menaquinone pool [27]. It is thought that electrons are transferred from the reduced quinone pool to adenosine phosphosulfate and sulfite via the membrane-bound respiratory complexes Qmo [28] and Dsr [29], respectively. A similar electron transfer chain for the oxidation of $\mathrm{H}_{2}$ with sulfate appears to be functional in D. retbaense: The uptake of $\mathrm{H}_{2}$ is probably catalyzed in this species by a heterodimeric NiFe- or $\mathrm{NiFeSe-hydrogenase} \mathrm{encoded} \mathrm{by} \mathrm{the} \mathrm{genes}$ Dret_0265 (hydB) and Dret_0266 (hydA). Six genes of the completed genome were annotated as cytochromes class III containing at least one domain with homology to a tetraheme cytochrome $c_{3}$. Electrons could be transferred from the reduced cytochrome c pool to menaquinone by a putative Mop complex (Dret_0270/Dret_0273) that is located in close proximity to the hydrogenase genes. 
A membrane-bound Qmo complex (Dret_1963, Dret_1964, and Dret_1965) was also identified adjacent to the genes of the dissimilatory adenylyl sulfate reductase $(a p r A B)$. Likewise, genes encoding the five subunits DsrMKJOP complex (Dret_0235/Dret_0239) were found close to genes of the $\alpha$ and $\beta$ subunits of the dissimilatory sulfite reductase $(d s r A B)$. Hence, it appears that the pro- posed organization of the electron transfer chain from $\mathrm{H}_{2}$ to sulfate seems to be conserved not only in species of the genus Desulfovibrio, but also in other sulfate-reducing members of the Deltaproteobacteria. In Figure 4 an illustration of the hypothetical electron transfer chain in D. retbaense is given, which is mainly based on results previously obtained with $\mathrm{H}_{2}$-utilizing Desulfovibrio species.

Table 3. Genome Statistics

\begin{tabular}{lrr}
\hline Attribute & \multicolumn{1}{c}{ Value } & \% of Total \\
\hline Genome size (bp) & $2,909,567$ & $100.00 \%$ \\
DNA coding region (bp) & $2,510,084$ & $86.27 \%$ \\
DNA G+C content (bp) & $1,666,078$ & $57.33 \%$ \\
Number of replicons & 2 & \\
Extrachromosomal elements & 1 & \\
Total genes & 2,609 & $100.00 \%$ \\
RNA genes & 57 & $2.18 \%$ \\
rRNA operons & 2 & \\
Protein-coding genes & 2,552 & $97.82 \%$ \\
Pseudo genes & 29 & $1.11 \%$ \\
Genes with function prediction & 1,920 & $73.59 \%$ \\
Genes in paralog clusters & 266 & $10.20 \%$ \\
Genes assigned to COGs & 1,976 & $75.74 \%$ \\
Genes assigned Pfam domains & 1,968 & $75.43 \%$ \\
Genes with signal peptides & 456 & $17.48 \%$ \\
Genes with transmembrane helices & 634 & $24.30 \%$ \\
CRISPR repeats & 1 & \\
\hline
\end{tabular}

Several genes that are involved in the incomplete oxidation of lactate to acetate could be detected in the complete genome sequence. The transport of lactate in the cytoplasm is probably facilitated by a specific permease encoded by the gene Dret_1039. Following transport, lactate is oxidized to pyruvate by a putative L-lactate dehydrogenase (Dret_0157). Pyruvate is then oxidatively decarboxylated by a pyruvate ferredoxin oxidoreductase to acetyl-CoA. Interestingly, the gene Dret_1036 encoding a homodimeric pyruvate ferredoxin oxidoreductase is located in close proximity to the lactate permease gene and genes responsible for the substrate level phosphorylation of ADP to ATP via conversion of acetyl-CoA to acetate, i.e. phosphotransacetylase (Dret_1035) and acetate kinase (Dret_1034).

Besides substrate level phosphorylation, generation of ATP is also possible by the utilization of a chemiosmotic proton gradient through a F0F1 ATP synthase complex, which is encoded at two different sites of the genome. One gene cluster encodes the cytoplasmic F1 part along with the Bsubunit of the membrane-bound F0 complex (Dret_2211/Dret_2217), whereas the remaining F0 subunits A (Dret_2087) and C (Dret_2086) are encoded elsewhere.

\section{Intermediary carbon metabolism}

D. retbaense is not able to grow autotrophically with $\mathrm{CO}_{2}$ as carbon source and needs acetate or complex carbon sources for growth with $\mathrm{H}_{2}$ as energy source. Intermediary carbon compounds required as precursors for the biosynthesis of cellular components are probably synthesized by a partial reverse tricarboxylic acid (TCA) cycle. A possible pathway for the assimilation of acetate starts with the synthesis of pyruvate from acetylCoA through a carboxylating reaction catalyzed by 
Spring et al.

the pyruvate ferredoxin oxidoreductase (Dret_1036). Pyruvate can then be either activated to phosphoenolpyruvate by the enzyme pyruvate, water dikinase (Dret_0098) to enable gluconeogenesis or is further carboxylated to oxaloacetate by pyruvate carboxylase, which is encoded by two separate genes (Dret_0690 and Dret_1120). Alternatively, pyruvate can be also used for the synthesis of malate by malic enzyme (Dret_0778), which requires $\mathrm{NADP}^{+}$as cofactor. The remaining major precursors for anabolic reactions can then be produced starting from malate by reactions of the re- verse TCA cycle, involving the enzymes fumarase (Dret_1068, Dret_1069), fumarate reductase (Dret_1065, Dret_1066, and Dret_1067), succinylCoA synthetase (Dret_0545) and 2-oxoglutarate ferredoxin oxidoreductase (Dret_1400/Dret_1403). The important five carbon precursor 2-oxoglutarate could also be synthesized from citrate by aconitase (Dret_1771) and isocitrate dehydrogenase (Dret_0439). Genes encoding the enzymes ATP-citrate lyase or citrate synthase were not detected in the annotated genome sequence, so that a closing of the TCA cycle is apparently prevented.
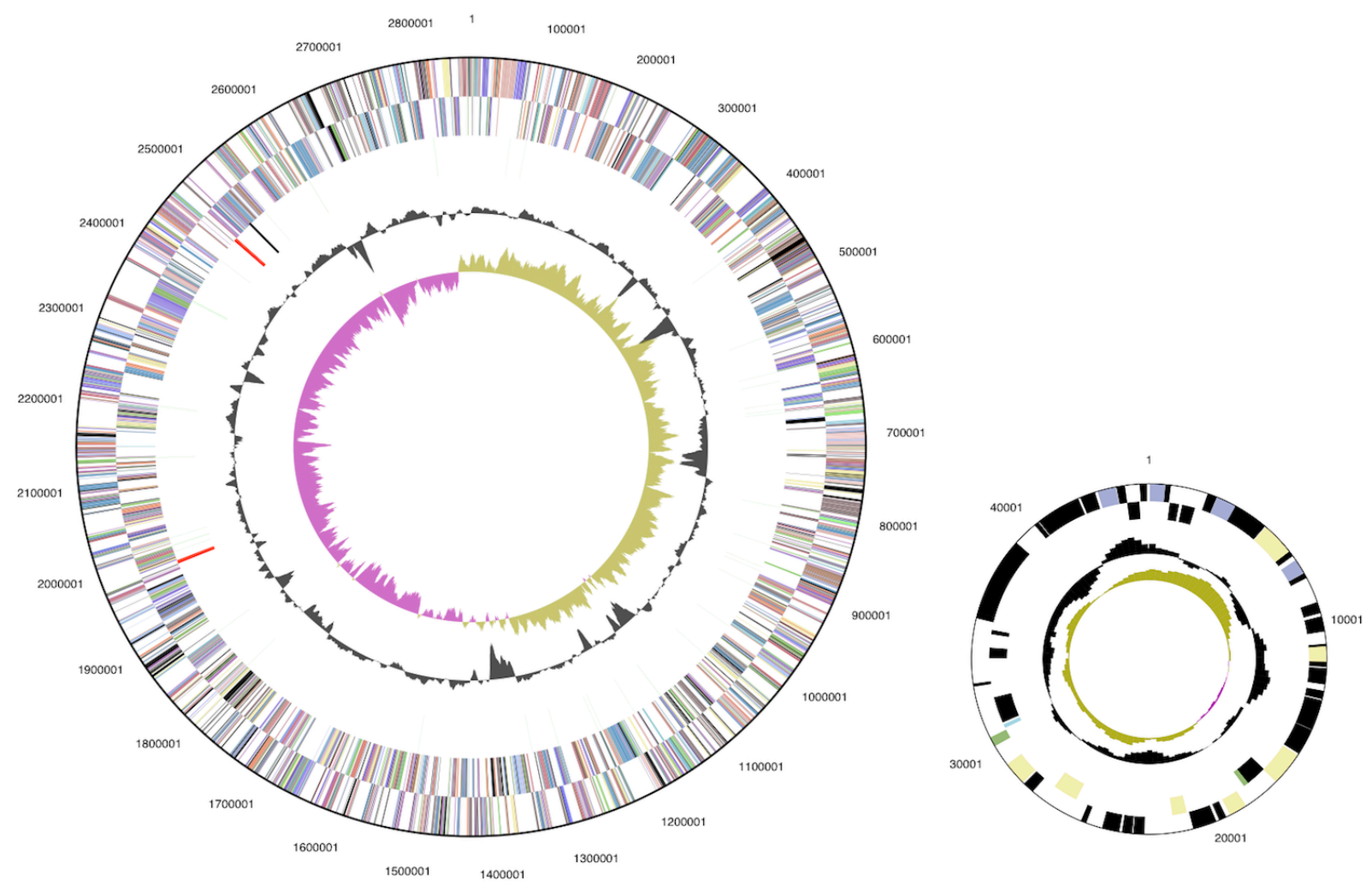

Figure 3. Graphical circular map of the genome. From outside to the center: Genes on forward strand (color by COG categories), Genes on reverse strand (color by COG categories), RNA genes (tRNAs green, rRNAs red, other RNAs black), GC content, GC skew.

\section{Defense against osmotic and oxidative stress}

Cells of $D$. retbaense inhabit saline environments and hence need appropriate protection against low water activity or varying salt concentrations. The accumulation of compatible solutes is a widespread strategy among microorganisms to protect against osmotic stress. In the distantly related moderately halophilic sulfate-reducing bacterium Desulfovibrio halophilus, the organic solutes trehalose and glycine betaine were identified as osmoprotectants [30]. In the genome of $D$. retbaense
DSM 5692 several genes could be detected that may be involved in the intracellular synthesis or accumulation of the above mentioned compatible solutes. For instance, the organic solute trehalose can be synthesized from UDP-D-glucose and alpha-D-glucose 6-phosphate by the enzymes trehalose-6-phosphate synthase (Dret_1902) and trehalose-6-phosphatase (Dret_1903). Alternatively, trehalose may be produced from the reserve carbohydrate glycogen, if the enzymes malto- 
oligosyltrehalose synthase (Dret_0039) and malto-oligosyltrehalose trehalohydrolase (Dret_0037) are expressed. On the other hand, the gene Dret_0035 encodes a trehalose synthase that can transform maltose directly into trehalose and vice versa, so that an excess of trehalose can be converted to glycogen again.

Table 4. Number of genes associated with the general COG functional categories

\begin{tabular}{lrrl}
\hline Code & value & \%age & Description \\
\hline J & 151 & 5.9 & Translation, ribosomal structure and biogenesis \\
A & 0 & 0.0 & RNA processing and modification \\
K & 94 & 3.7 & Transcription \\
L & 129 & 5.1 & Replication, recombination and repair \\
B & 2 & 0.1 & Chromatin structure and dynamics \\
D & 28 & 1.1 & Cell cycle control, mitosis and meiosis \\
Y & 0 & 0.0 & Nuclear structure \\
V & 21 & 0.8 & Defense mechanisms \\
T & 168 & 6.6 & Signal transduction mechanisms \\
M & 146 & 5.7 & Cell wall/membrane biogenesis \\
N & 74 & 2.9 & Cell motility \\
Z & 0 & 0.0 & Cytoskeleton \\
W & 0 & 0.0 & Extracellular structures \\
U & 82 & 3.2 & Intracellular trafficking and secretion \\
O & 100 & 3.9 & Posttranslational modification, protein turnover, chaperones \\
C & 191 & 7.5 & Energy production and conversion \\
G & 101 & 4.0 & Carbohydrate transport and metabolism \\
E & 188 & 7.4 & Amino acid transport and metabolism \\
F & 53 & 2.1 & Nucleotide transport and metabolism \\
H & 109 & 4.3 & Coenzyme transport and metabolism \\
I & 43 & 1.7 & Lipid transport and metabolism \\
P & 95 & 3.7 & Inorganic ion transport and metabolism \\
Q & 20 & 0.8 & Secondary metabolites biosynthesis, transport and catabolism \\
R & 212 & 8.3 & General function prediction only \\
S & 152 & 6.0 & Function unknown \\
- & 633 & 24.8 & Not in COGs \\
\hline
\end{tabular}

The second osmotic solute in $D$. retbaense appears to be glycine betaine, which can be accumulated in two different ways: The most efficient way in terms of energy represents the uptake from the environment. Glycine betaine is produced by many cyanobacteria or halophilic anoxygenic photosynthetic bacteria and is released continuously in the environment by excretion or cell lysis. The genome of $D$. retbaense DSM 5692 contains two distinct gene clusters (Dret_0768/Dret_0771 and Dret_22771/Dret_22773) that could encode high affinity $\mathrm{ABC}$ transporters for the uptake of glycine betaine. Several separate genes encoding periplasmic glycine betaine binding proteins were also found and could have a function in the regulation of genes in response to the presence of glycine betaine in the environment. An alternative route for the accumulation of glycine betaine is based on the uptake of choline. This quaternary amine is an essential component of eukaryotic cell membranes and hence ubiquitous in most environments. Two genes were found that encode putative choline transporters, Dret_1055 and Dret_2376. Following transport to the cytoplasm, choline could then be oxidized to glycine betaine by the enzymes choline dehydrogenase (Dret_0130) and betaine aldehyde dehydrogenase (Dret_0129). 


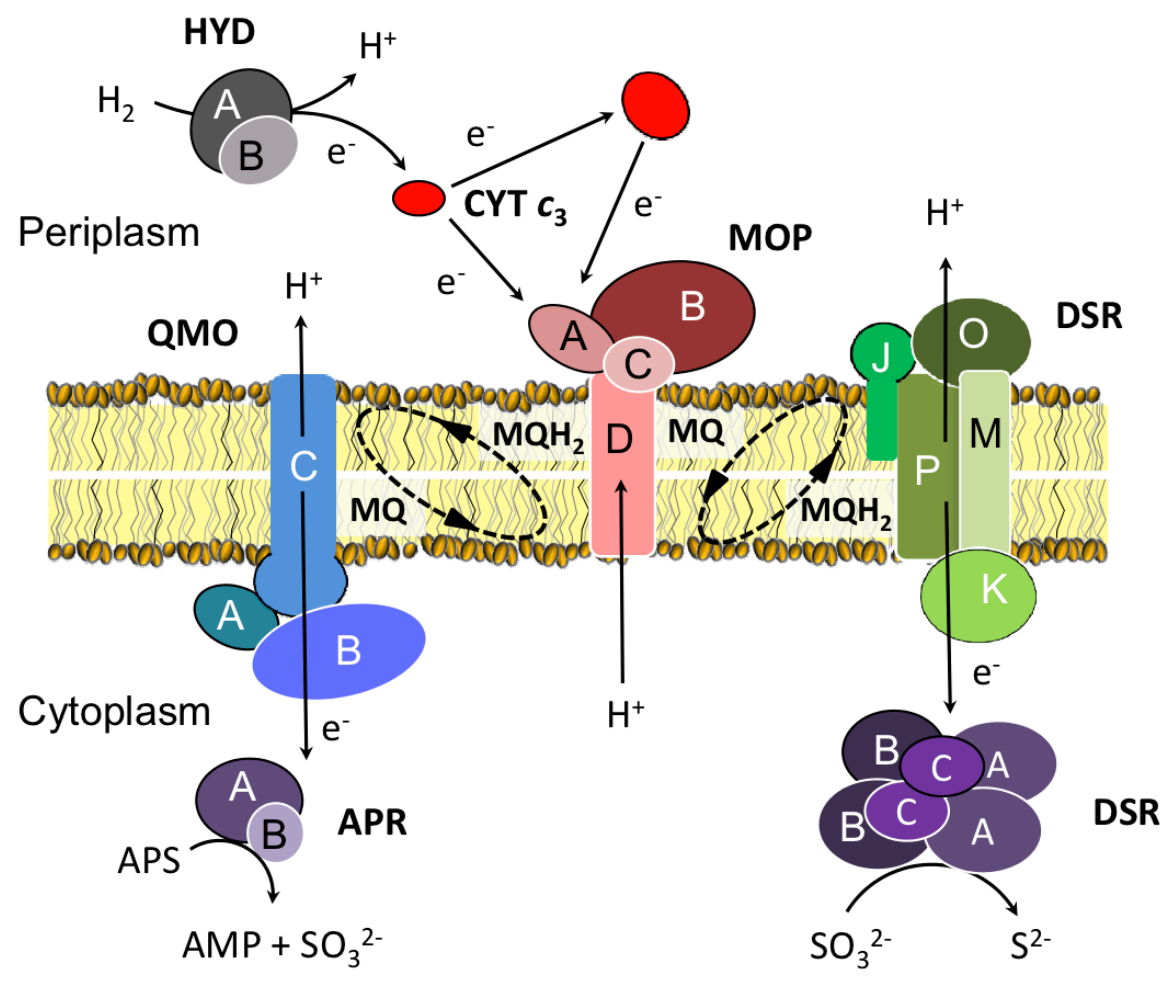

Figure 4. Proposed organization of the electron transfer chain in D. retbaense with $\mathrm{H}_{2}$ as electron donor and sulfate as electron acceptor. Gene products are designated according to the information given in Supplementary Table 1. Subunits of multiprotein complexes are labeled with capital letters. Abbreviations: APS, adenosine-5'-phosphosulfate; AMP, adenosine monophosphate; $\mathrm{MQ}$, menaquinone; $\mathrm{MQH} 2$, dihydromenaquinone.

The sensitivity of the obligately anaerobic species D. retbaense to oxygen exposure has not been analyzed in detail, but it can be assumed that it is quite moderate as in most other studied sulfatereducing bacteria [31]. A close inspection of the annotated genome sequence revealed a complex network of antioxidant proteins protecting cells of this species against oxidative stress. Aerobic respiration was identified as one principal mechanism for the detoxification of oxygen in Gramnegative sulfate-reducers [32]. In D. retbaense DSM 5692, genes for the two subunits of a cytochrome $b d$ quinol oxidase (Dret_0135 and Dret_0136) were identified. This type of oxidase is the most common terminal oxidase among Gramnegative sulfate-reducers and characterized by a high-affinity to oxygen [32,33]. For the detoxification of reactive oxygen species that emerge from the contact of oxygen with cellular redox enzymes several protection systems seem to be present. A di-heme cytochrome $c$ peroxidase (Dret_1885) that is probably localized in the periplasmic space [34] is able to reduce $\mathrm{H}_{2} \mathrm{O}_{2}$ to water, whereas a catalase (Dret_1236) produces oxygen from the inactivation of $\mathrm{H}_{2} \mathrm{O}_{2}$. On the other hand, in the cytoplasm multiprotein complexes containing rubredoxins (Dret_0886, Dret_0139), rubrerythrins (Dret_0191, Dret_1205, Dret_1644, Dret_2310) and desulfoferrodoxin (Dret_0140) could establish electron transfer systems for the reduction of superoxide radicals and $\mathrm{H}_{2} \mathrm{O}_{2}[35,36]$. Finally, cellular proteins and lipids that became damaged by reactive oxygen species could be repaired by a methionine sulfoxide reductase (Dret_2264), peroxiredoxin (Dret_2393) and an alkylhydroperoxidase (Dret_1223).

Thus, based on the results of the genome analysis it seems that this species is very well adapted to frequent changes in salinity and redox conditions in its natural environment, the sediments of hypersaline lakes. 


\section{Acknowledgements}

We would like to gratefully acknowledge the help of Susanne Schneider (DSMZ) for DNA extraction and quality analysis. This work was performed under the auspices of the US Department of Energy's Office of Science, Biological and Environmental Research Program, and by the University of California, Lawrence Berkeley

\section{References}

1. Ollivier B, Hatchikian CE, Prensier G, Guezennec J, Garcia JL. Desulfohalobium retbaense gen. nov., sp. nov., a halophilic sulfate-reducing bacterium from sediments of a hypersaline lake in Senegal. Int / Syst Bacteriol 1991; 41:74-81.

2. Kjeldsen KU, Loy A, Jakobsen TF, Thomsen TR, Wagner M, Ingvorsen K. Diversity of sulfatereducing bacteria from an extreme hypersaline sediment, Great Salt Lake (Utah). FEMS Microbiol Ecol 2007; 60:287-298. doi:10.1111/j.15746941.2007.00288.x

3. Oren A. Bioenergetic aspects of halophilism. Microbiol Mol Biol Rev 1999; 63:334-348.

4. Copeland A, Spring S, Göker M, Schneider S, Lapidus A, Glavina Del Rio T, Tice H, Cheng JF, Lucas $\mathrm{S}$, Chen F, et al. Complete genome sequence of Desulfomicrobium baculatum type strain $\left(\mathrm{X}^{\mathrm{T}}\right)$. Stand Genomic Sci 2009; 1:29-37. doi:10.4056/sigs.13134

5. Kuever J, Rainey FA, Widdel F. Family III. Desulfohalobiaceae fam. nov. In Bergey's Manual of Systematic Bacteriology 2nd ed. (Brenner DJ, Krieg NR, Staley JT and Garrity GM, eds.), Springer Verlag, New York 2005; 2:948-949.

6. Lee C, Grasso C, Sharlow MF. Multiple sequence alignment using partial order graphs. Bioinformatics 2002; 18:452-464. doi:10.1093/bioinformatics/18.3.452

7. Castresana J. Selection of conserved blocks from multiple alignments for their use in phylogenetic analysis. Mol Biol Evol 2000; 17:540-552.

8. Stamatakis A, Hoover P, Rougemont J. A rapid bootstrap algorithm for the RAxML web-servers. Syst Biol 2008; 57:758-771. doi:10.1080/10635150802429642

9. Liolios K, Mavromatis K, Tavernarakis N, Kyrpides NC. The Genomes OnLine Database (GOLD) in 2007: status of genomic and metagenomic projects and their associated metadata. Nucleic Acids Res 2008; 36:D475-D479. doi:10.1093/nar/gkm884
National Laboratory under contract No. DE-AC0205CH11231, Lawrence Livermore National Laboratory under Contract No. DE-AC52-07NA27344, and Los Alamos National Laboratory under contract. German Research Foundation (DFG) supported DSMZ under INST 599/1-1.

10. Jakobsen TF, Kjeldsen KU, Ingvorsen K. Desulfohalobium utahense sp. nov., a moderately halophilic sulfate-reducing bacterium isolated from Great Salt Lake. Int I Syst Evol Microbiol 2006; 56:2063-2069. doi:10.1099/ijs.0.64323-0

11. Field D, Garrity G, Gray T, Morrison N, Selengut J, Sterk P, Tatusova T, Tompson N, Allen MJ, Angiuoli SV, et al. Towards a richer description of our complete collection of genomes and metagenomes: the "Minimum Information about a Genome Sequence" (MIGS) specification. Nat Biotechnol 2008; 26:541-547. doi:10.1038/nbt1360

12. Woese CR, Kandler O, Wheelis ML. Towards a natural system of organisms: proposal for the domains Archaea, Bacteria, and Eucarya. Proc Natl Acad Sci USA 1990; 87:4576-4579. doi:10.1073/pnas.87.12.4576

13. Garrity GM, Holt JG. Taxonomic Outline of the Archaea and Bacteria. Bergey's Manual of Systematic Bacteriology 2nd ed. (Boone DR and Castenholz RW, eds.), Springer-Verlag, New York $2001 ; 1: 155-166$.

14. Kuever J, Rainey FA, Widdel F. Order II. Desulfovibrionales ord. nov. In: Garrity GM, Brenner DJ, Krieg NR, Staley JT (eds), Bergey's Manual of Systematic Bacteriology, Second Edition, Volume 2, Part C, Springer, New York, 2005, p. 925-926.

15. Editor L. Validation List No. 107. List of new names and new combinations previously effectively, but not validly, published. Int I Syst Evol Microbiol 2006; 56:1-6. doi:10.1099/ijs.0.64188$\underline{0}$

16. Anonymous. Biological Agents: Technical rules for biological agents (TRBA 466) www.baua.de

17. Ashburner M, Ball CA, Blake JA, Botstein D, Butler H, Cherry JM, Davis AP, Dolinski K, Dwight SS, Eppig JT, et al. Gene ontology: tool for the unification of biology. Nat Genet 2000; 25:25-29. doi:10.1038/75556

18. Belyakova EV, Rozanova EP, Borzenkov IA, Tourova TP, Pusheva MA, Lysenko AM, Kolganova TV. The new facultatively chemolithoautotrophic, 
Spring et al.

moderately halophilic, sulfate-reducing bacterium Desulfovermiculus halophilus gen. nov., sp. nov., isolated from an oil field. [english translation of Mikrobiologiya]. Microbiology 2006; 75:161-171. doi:10.1134/S0026261706020093

19. Wu D, Hugenholtz P, Mavromatis K, Pukall R, Dalin E, Ivanova N, Kunin V, Goodwin L, Wu M, Tindall BJ, et al. A phylogeny-driven genomic encyclopedia of Bacteria and Archaea. Nature 2009; 462:1056-1060. doi:10.1038/nature08656

20. List of growth media used at DSMZ: http://www.dsmz.de/microorganisms/ media_list.php.

21. Han CS, Chain P. Finishing repeat regions automatically with Dupfinisher. In: Proceeding of the 2006 international conference on bioinformatics \& computational biology. Hamid R Arabnia \& Homayoun Valafar (eds), CSREA Press. June 2629, 2006:141-146.

22. Sims D, Brettin T, Detter JC, Han C, Lapidus A, Copeland A, Glavina Del Rio T, Nolan M, Chen $\mathrm{F}$, Susan L, et al. Complete genome sequence of Kytococcus sedentarius type strain $\left(541^{\mathrm{T}}\right)$. Stand Genomic Sci 2009; 1:12-20. doi:10.4056/sigs.761

23. Anonymous. Prodigal Prokaryotic Dynamic Programming Genefinding Algorithm. Oak Ridge National Laboratory and University of Tennessee 2009 http://compbio.ornl.gov/prodigal

24. Pati A, Ivanova N, Mikhailova N, Ovchinikova G, Hooper SD, Lykidis A, Kyrpides NC. GenePRIMP: A Gene Prediction Improvement Pipeline for microbial genomes. (Submitted).

25. Markowitz VM, Mavromatis K, Ivanova NN, Chen IMA, Chu K, Kyrpides NC. Expert Review of Functional Annotations for Microbial Genomes. Bioinformatics 2009; 25:2271-2278. doi:10.1093/bioinformatics/btp393

26. Matias PM, Pereira IAC, Soares CM, Carrondo MA. Sulphate respiration from hydrogen in Desulfovibrio bacteria: a structural biology overview. Prog Biophys Mol Biol 2005; 89:292-329. doi:10.1016/j.pbiomolbio.2004.11.003

27. Li X, Luo Q, Wofford NQ, Keller KL, McInerney MM, Wall JD, Krumholz LR. A molybdopterin oxidoreductase is involved in $\mathrm{H}_{2}$ oxidation in Desulfovibrio desulfuricans G20. I Bacteriol 2009; 191:2675-2682. doi:10.1128/JB.01814-08

28. Pires RH, Lourenco Al, Morais F, Teixeira M, Xavier AV, Saraiva LM, Pereira IAC. A novel membrane-bound respiratory complex from Desulfovibrio desulfuricans ATCC 27774. Biochim Biophys Acta 2003; 1605:67-82. doi:10.1016/S0005-2728(03)00065-3

29. Pires RH, Venceslau SS, Morais F, Teixeira M, Xavier AV, Pereira IAC. Characterization of the Desulfovibrio desulfuricans ATCC 27774 DsrMKJOP complex-a membrane-bound redox complex involved in the sulfate respiratory pathway. Biochemistry (Mosc) 2006; 45:249-262. doi:10.1021/bi0515265

30. Welsh DT, Lindsay YE, Caumette P, Herbert RA, Hannan J. Identification of trehalose and glycine betaine as compatible solutes in the moderately halophilic sulfate reducing bacterium Desulfovibrio halophilus. FEMS Microbiol Lett 1996;

140:203-207. doi:10.1111/j.15746968.1996.tb08337.x

31. Marschall C, Frenzel P, Cypionka H. Influence of oxygen on sulfate reduction and growth of sulfate-reducing bacteria. Arch Microbiol 1993; 159:168-173. doi:10.1007/BF00250278

32. Cypionka H. Oxygen respiration by Desulfovibrio species. Annu Rev Microbiol 2000; 54:827-848. doi:10.1146/annurev.micro.54.1.827

33. Spring S, Lapidus A, Schröder M, Gleim D, Sims D, Meincke L, Glavina Del Rio T, Tice H, Copeland $\mathrm{A}$, Cheng JF, et al. Complete genome sequence of Desulfotomaculum acetoxidans type strain $\left(5575^{\top}\right)$. Stand Genomic Sci 2009; 1:242253. doi:10.4056/sigs.39508

34. Goodhew CF, Wilson IBH, Hunter DJB, Pettigrew GW. The cellular location and specificity of bacterial cytochrome c peroxidases. Biochem / 1990; 271:707-712.

35. Lumppio HL, Shenvi NV, Summers AO, Voordouw G, Kurtz DM, Jr. Rubrerythrin and rubredoxin oxidoreductase in Desulfovibrio vulgaris: a novel oxidative stress protection system. I Bacteriol 2001; 183:101-108. doi:10.1128//B.183.1.101-108.2001

36. Kawasaki S, Sakai Y, Takahashi T, Suzuki I, Niimura $\mathrm{Y} . \mathrm{O}_{2}$ and reactive oxygen species detoxification complex, composed of $\mathrm{O}_{2}$-responsive $\mathrm{NADH}$ :rubredoxin oxidoreductase-flavoprotein A2-desulfoferrodoxin operon enzymes, rubperoxin, and rubredoxin, in Clostridium acetobutylicum. Appl Environ Microbiol 2009; 75:1021-1029. doi:10.1128/AEM.01425-08 